\title{
Management of advanced vaginal melanoma: a case report and review of the literature
}

\author{
Taylor Orellana ${ }^{1}$, Sushil Beriwal ${ }^{2}$, Joseph L Kelley ${ }^{1}$, Robert P Edwards ${ }^{1}$, Paniti Sukumvanich ${ }^{2}$ and Alexander B Olawaiye ${ }^{1 *}$ \\ ${ }^{1}$ Department of Obstetrics, Gynecology, Magee-Women's Hospital of UPMC, 300 Halket Street, Pittsburgh, PA 15213, USA \\ ${ }^{2}$ Department of Radiation Oncology, Magee-Women's Hospital of UPMC, 300 Halket Street, Pittsburgh, PA 15213, USA
}

\begin{abstract}
Vaginal melanoma is the second most common primary vaginal malignancy and is associated with very poor survival outcomes. Surgery is the mainstay of treatment for vaginal melanoma, whereas primary radiation therapy is utilized much less commonly. This case report details the clinical course of a patient with locally advanced vaginal melanoma who demonstrated a complete and durable reponse to therapy after treatment with external beam radiation therapy without chemosensitization and vaginal brachytherapy alone. Additionally, this report includes a summary of the available literature with regard to treatment methods for primary vaginal melanoma, including both surgical management and radiation therapy. Based on our patient's response to therapy and our findings on literature review, we advocate for continued investigation into the role of radiation therapy in the treatment of primary vaginal melanoma.
\end{abstract}

\section{Introduction}

Vaginal melanoma is the second most common histologic subtype of primary vaginal cancer, accounting for approximately $4 \%$ of all vaginal malignancies [1]. In women, genital tract melanomas most commonly affect the vulva, whereas vaginal melanomas comprise only $19.8 \%$ of cases [2]. The prognosis of vaginal melanoma is extremely poor. While cutaneous melanoma and vulvar melanoma are characterized by $89 \%$ and $60 \% 5$-year relative survival rates, respectively, the 5-year relative survival rate for primary vaginal melanoma is $14-25 \%[1,3,4]$. Surgery, when feasible, is often recommended as the standard of treatment for vaginal melanoma, and is the most frequently used primary therapy described in the literature [5-7]. In one large review of 805 cases of vaginal melanoma, only $5 \%$ of patients were treated with radiotherapy alone [5].

This case report describes the treatment of locally advanced vaginal melanoma with primary definitive external beam radiation therapy without chemosensitization and vaginal brachytherapy with Iridium-192.

\section{Case}

The patient is an 84-year-old gravida 5 para 5 who first presented to care in December 2013 with vaginal bleeding. She was evaluated by her primary gynecologist and was diagnosed with a vaginal polyp. On a follow-up examination, a second lesion was noted. Due to concern for malignancy, she was referred to the Gynecologic Oncology Division at Magee-Womens Hospital for consultation, however prior to her appointment, she presented to the Emergency Department due to ongoing vaginal bleeding. The patient had a history of normal Pap tests and went through menopause at age 50 . She denied any prior history of postmenopausal bleeding. On physical exam in the Emergency Department, she was noted to have two masses originating internal to the vaginal introitus. The first mass was slightly to the right of the urethra and was hyperpigmented and polypoid with an identifiable stalk. The second mass was located on the posterior vaginal wall and was noted to be vascular and polypoid with an identifiable stalk. The remainder of her pelvic exam, including the cervix, was normal. The posterior vaginal lesion was biopsied and sent for pathologic examination with histologic findings of a high-grade neoplasm with necrosis, and immunohistochemical testing positive for WT1, S-100, HMB-45, Melan-A, and Tyrosinase. Additionally, a C-KIT gene mutation analysis was performed and was positive for a K642E mutation. A diagnosis of malignant melanoma was made based on these findings. A pelvic MRI (Figure 1) and full body PET/CT (Figure 2 and Figure 3 ) were performed and notable for findings of a hypermetabolic vulvar lesion anteriorly in the midline measuring approximately $2 \mathrm{~cm}$, as well as mural thickening and edema of the adjacent anterior vagina with diffuse moderate FDG uptake. No metastatic disease or lymph node involvement were demonstrated on imaging. On physical examination in the office, a $3 \times 2 \mathrm{~cm}$ tumor was noted in close proximity to the urethral meatus. Notably, the tumor was confined to the vagina and did not involve the vulva. Based on these findings, the patient's clinical staging was determined to be FIGO stage III (T3 N0 M0). She was not a candidate for surgical excision due to her age and the location/extent of disease. She was therefore referred to Radiation Oncology. Unfortunately, she was unable to make her Radiation Oncology visit at a short interval, and by the time she was seen 6 weeks later, her tumor was noted to have nearly doubled in size. The patient underwent definitive radiation therapy with a combination of external beam radiation therapy (EBRT) and brachytherapy. EBRT was delivered to the primary site and to low lying pelvic and inguinal nodes. The planned dose was $40 \mathrm{~Gy}$ at 2.5

${ }^{\star}$ Correspondence to: Alexander B Olawaiye, Department of Obstetrics and Gynecology, Division of Gynecologic Oncology, Magee-Women's Hospital of UPMC, 300 Halket Street, Pittsburgh, PA 15213, USA, E-mail: olawaiyea@ upmc.edu

Key words: melanoma, primary, radiation, treatment, vaginal

Received: August 08, 2020; Accepted: September 18, 2020; Published: September 24, 2020 
Gy per fraction, however treatment was stopped at 32.5 Gy due to a vulvar reaction. The patient then received HDR brachytherapy boost of $30 \mathrm{~Gy}$ in 5 fractions with an MRI-based plan using multichannel cylinder and brachytherapy (Figure 4). The total dose to the primary target was equivalent to $70 \mathrm{~Gy}$ at $2 \mathrm{~Gy}$ per fraction, while critical organs like the rectum and bladder received $42 \mathrm{~Gy}$ and $50 \mathrm{~Gy}$, respectively. After radiation therapy, the patient experienced intermittent diarrhea that was controlled with medical management. At her follow-up examination in the office approximately 5 weeks after completion of brachytherapy, she had no evidence of disease. The patient continued to follow-up at regular intervals with Medical Oncology and her primary OB/GYN, however she was lost to Gynecologic Oncology follow-up for four years. When she represented to the Gynecologic Oncology office in April 2018, the patient had no clinical or radiologic evidence of disease. At the time of her most recent follow-up in November 2019, the patient remained without evidence of disease on clinical exam and MRI.

\section{Discussion}

Vaginal melanoma is rare, occurring at an age-adjusted rate of 0.3 per million per year [2]. In a recent Consensus Review from the Gynecologic Cancer InterGroup on vulvovaginal melanomas [6],

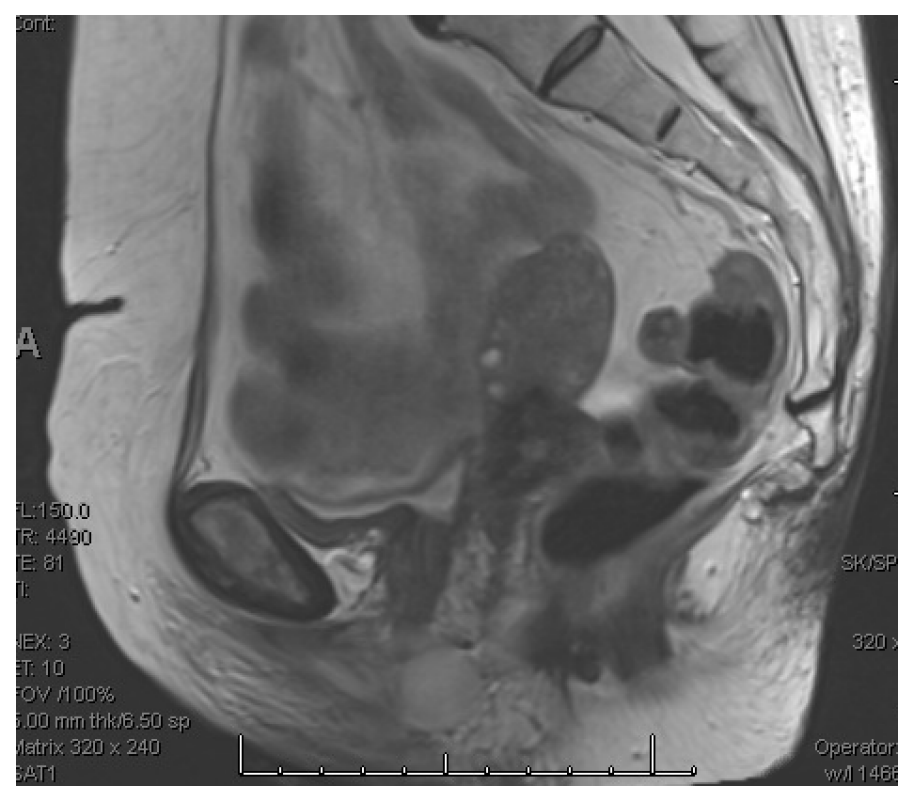

Figure 1. Pelvic MRI (sagittal view)

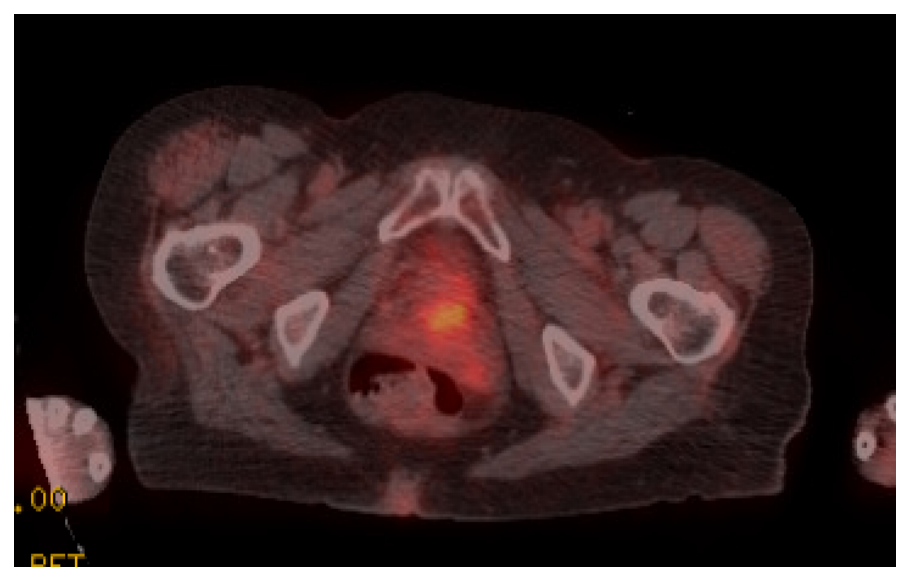

Figure 2. PET/CT (axial view)

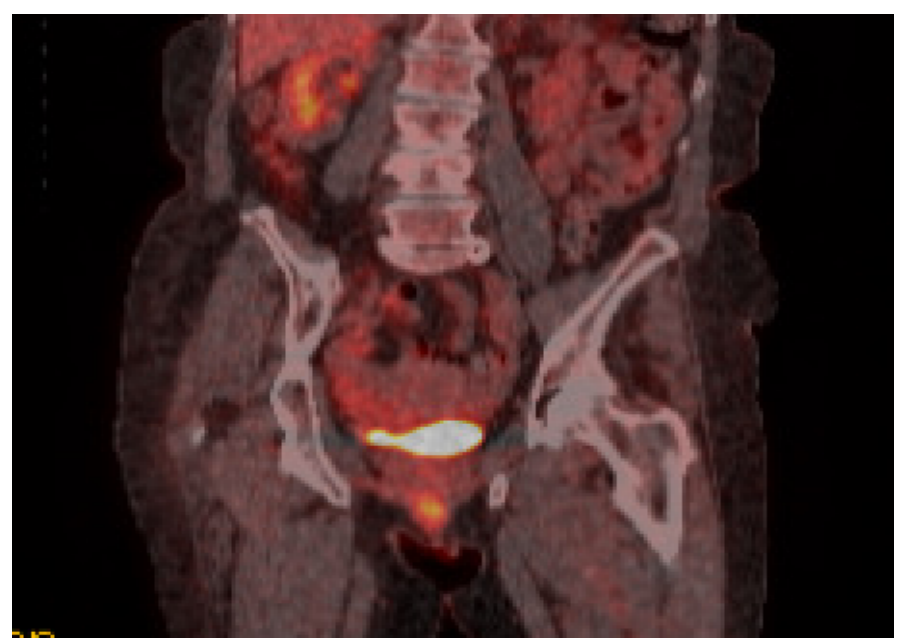

Figure 3. PET/CT (coronal view)

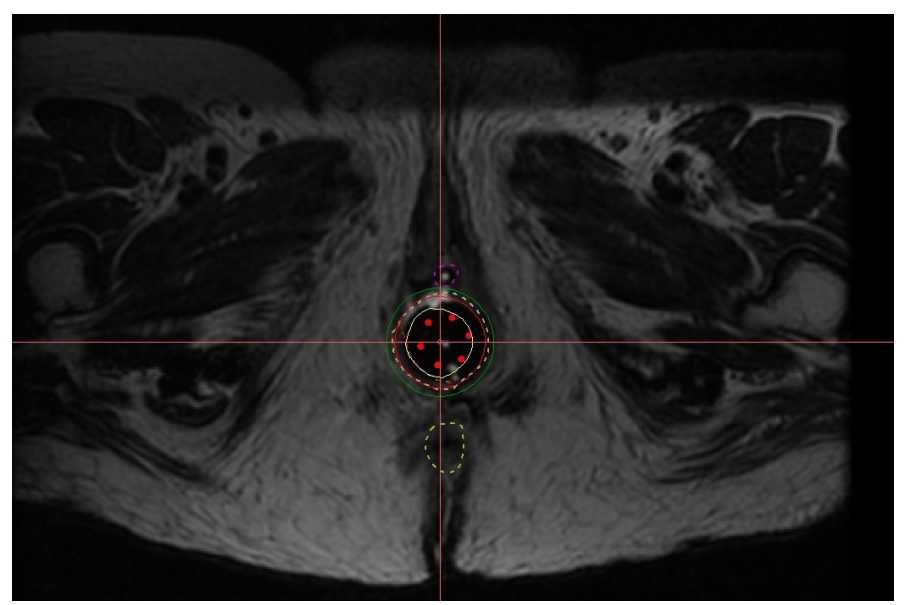

Figure 4. T2 sequence of MRI for vaginal brachytherapy with multichannel cylinder in place

as well as a recent review of the literature in Gynecologic Oncology [7], surgery is recommended as the preferred primary treatment for vulvovaginal melanomas. Radiation therapy has been recommended as a consideration for adjunctive therapy after surgery and for advanced or recurrent disease that is not amenable to surgical resection [6,7]. Review of the literature suggests that radiation alone for treatment of vaginal melanoma is not routinely used, providing limited opportunity to study patients' outcomes. In an analysis of 805 cases of vaginal melanoma, radiation therapy alone was used in only $5 \%$ of cases, while surgical resection with or without other adjuvant therapies was used in $89 \%$ of cases [5]. Several retrospective reviews of the treatment of vaginal melanoma have demonstrated statistically significant differences in outcomes when comparing surgery to radiation therapy $[8,9,10]$. In a study of 37 patients treated for stage I primary vaginal melanoma at MD Anderson, median overall survival (OS) for patients who underwent treatment with chemotherapy, radiation, or a combination of both was 8.7 months, which was significantly worse than for patients who underwent primary surgical excision or pelvic exenteration (median OS of 24.3 months and 34.4 months, respectively) [8]. Similarly, in a 2013 study of 201 patients with vaginal melanoma identified in the Surveillance, Epidemiology, and End Result (SEER) registry, OS was significantly higher for patients who underwent surgical treatment compared to those who did not receive surgery, and none of the patients who were treated with radiation alone survived longer than 3 
years [9]. Additionally, in a retrospective analysis of 35 patients from Memorial Sloan-Kettering, primary surgical therapy was associated with a median OS of 25 months, compared to just 13 months when patients underwent primary radiation therapy [10]. This difference was statistically significant, even when patients with surgically unresectable disease were excluded [10].

However, regardless of treatment type, the prognosis of vaginal melanoma is poor, with a median OS of 14 to 19 months and a 5-year relative survival rate of $14-25 \%$ [1,3,4,9]. Our patient demonstrates a rare instance of a complete and durable response of locally advanced vaginal melanoma to primary radiotherapy alone. Similar findings have only been described in a limited number of case reports and case series [11-13]. Petru et al. [11] reported long-term survival of 3 patients with vaginal melanoma, including 2 patients who underwent primary radiation therapy with external beam radiotherapy and vaginal brachytherapy and 1 patient who underwent adjuvant external beam radiotherapy. All three patients survived for at least 5 years, however, the radiotherapy regimens differed amongst the patients and all ultimately developed recurrent disease [11]. McGuire, Frank, and Eifel [12] reported a case of complete resolution of locally recurrent vaginal melanoma after a combination of EBRT and interstitial palladium implant. The patient was diagnosed with diffuse metastatic disease 16 months later without a local recurrence [12]. Finally, in a case series from the University of Virginia, the authors found that 2 patients treated with wide local excision followed by high-dose fractionation vaginal teletherapy demonstrated a durable locoregional response to therapy [13].

The case presented here advocates for further study into radiation therapy for primary treatment of vaginal melanoma and consideration of integration of image-based planning to reduce treatment morbidities. This case also emphasizes the importance of continuing to explore alternative and adjunctive therapies for this rare and aggressive disease.

\section{Acknowlegements}

None

\section{Funding information}

No funding was solicited or required for this work

\section{Conflicts of interest}

The authors declare that they have no competing interests.

\section{References}

1. Creasman WT, Phillips JLP, Menck HR (1998) The National Cancer Data Base report on cancer of the vagina. Cancer 83: 1033-1040. [Crossref]

2. McLaughlin CC, Wu XC, Jemal A, Martin HJ, Roche LM, et al. (2005) Incidence of noncutaneous melanomas in the U.S. Cancer 103: 1000-1007. [Crossref]

3. Bishop KD, Olszewski AJ (2014) Epidemiology and survival outcomes of ocular and mucosal melanomas: A population-based analysis. Int J Cancer 134: 2961-2971. [Crossref]

4. Weinstock MA (1994) Malignant melanoma of the vulva and vagina in the United States: patterns of incidence and population-based estimates of survival. Am J Obstet Gynecol 171: 1225-1230.

5. Rapi V, Dogan A, Schultheis B, Hartmann F, Rezniczek GA, et al. (2017) Melanoma of the vagina: case report and systemic review of the literature. Anticancer Res 37 6911-6920

6. Leitao MM, Cheng X, Hamilton AL, Siddiqui NA, Jurgenliemk-Schulz I, et al (2014) Gynecologic Cancer Inter Group (GCIG) consensus review for vulvovaginal melanomas. Int J Gynecol Cancer 24: S117-S122. [Crossref]

7. Gadducci A, Carinelli S, Guerrieri MD, Aletti GD (2018) Melanoma of the lower genital tract: Prognostic factors and treatment modalities. Gynecol Oncol 150: 180-189.

8. Frumovitz M, Etchepareborda M, Sun CC, Soliman PT, Eifel PJ, et al. (2010) Primary malignant melanoma of the vagina. Obstet Gynecol 116: 1358-1365.

9. Kirschner AN, Kidd EA, Dewees T, Perkins SM (2013) Treatment approach and outcomes of vaginal melanoma. Int J Gynecol Cancer 23: 1484-1489.

10. Miner TJ, Delgado R, Zeisler J, Busam K, Alektiar K, et al. (2004) Primary vaginal melanoma: a critical analysis of therapy. Ann Surg Oncol 11: 34-39.

11. Petru E, Nagele F, Czerwenka K, Graf AH, Lax S, et al. (1998) Primary malignant melanoma of the vagina: long-term remission following radiation therapy. Gynecol Oncol 70: 23-26. [Crossref]

12. McGuire SE, Frank SJ, Eifel PJ (2008) Treatment of recurrent vaginal melanoma with external beam radiation therapy and palladium-103 brachytherapy. Brachytherapy 7 : 359-363. [Crossref]

13. Irvin WP Jr, Bliss SA, Rice LW, Taylor PT Jr, Andersen WA (1998) Malignant melanoma of the vagina and locoregional control: radical surgery revisited. Gynecol Oncol 71: 476-480. [Crossref]

Copyright: (C2020 Orellana T. This is an open-access article distributed under the terms of the Creative Commons Attribution License, which permits unrestricted use, distribution, and reproduction in any medium, provided the original author and source are credited. 